\title{
Bagazo de cervecería como ingrediente en el desarrollo de panificados. Impacto del rotulado en la intención de compra y aceptabilidad
}

\section{Brewer's spent grain as an ingredient in the development of bakeries. Impact of labeling on purchase intention and acceptability}

Arcia, Patricia (1, 2) Curutchet, Ana (2); Cozzano, Sonia (2); Rodríguez, Santiago (2)

(1) Latitud - Fundación LATU, Montevideo, Uruguay

(2) Departamento de Ciencia y Tecnología de Alimentos, Facultad de Ingeniería y Tecnologías, UCU, Montevideo, Uruguay

Contacto: parcia@latitud.org.uy

RECIBIDO: 19/4/2018 - APROBADO: 18/9/2018

\begin{abstract}
Resumen
El objetivo de este trabajo fue revalorizar el residuo generado por la industria cervecera como un nuevo ingrediente rico en fibra y proteínas para el desarrollo de panes. Se evaluó el efecto de la información nutricional a presentar en forma de etiquetado frontal de octógonos en la aceptabilidad e intención de compra de los productos desarrollados, contemplando el claim "fuente de fibra" y la advertencia por "EXCESO DE SODIO". Se elaboraron panes con BSG (Brewer's Spent Grain), cumpliendo el claim "fuente de fibra", panes enriquecidos con fibra y panes de referencia sin incorporación de BSG. Las pruebas de aceptabilidad sensorial realizadas demuestran que el pan "fuente de fibra" presenta un valor significativamente inferior al pan de referencia y al pan enriquecido con fibra, que no presentan diferencias significativas entre sí. Tras las pruebas de aceptabilidad e intención de compra con información nutricional, se observó que la presencia de la advertencia "EXCESO DE SODIO" representada en el envase disminuye la intención de compra de los consumidores por panes con ese mensaje, mientras que la presencia del claim "fuente de fibra" lo aumentaría. Es una oportunidad para la industria, que debería focalizar esfuerzos hacia la reformulación y/o desarrollo de productos aceptables.

Palabras clave: subproducto de cervecería, fibra, pan, etiquetado frontal.
\end{abstract}

\begin{abstract}
The objective of this work was to revalue the waste generated by the brewing industry as a new ingredient rich in fibre and proteins for the development of bread. The effect of nutritional warnings used as front of pack nutritional information on the acceptability and purchase intention of the developed products were evaluated considering the claim "fibre source" and sodium warning. Breads were made with BSG (Brewer's spent grain), complying the claim "source of fibre", bread enriched with fibre and bread without incorporation of BSG. Acceptability test among them showed that bread "source of fibre" presented a value significantly lower than the bread without incorporation of BSG and bread enriched with fibre, which did not present significant differences between them. In the case of acceptability and purchase intention evaluation considering nutritional information, it was observed that the presence of warning "excess salt" represented on the packaging decreases the intention of consumers to buy that kind of product, while the presence of the claim "fibre source" would increase it. It is an opportunity for the industry, who should focus efforts towards the reformulation and/or development of acceptable products.

Keywords: Brewer's spent grain, fibre, bread, labeling.
\end{abstract}

\section{Introducción}

La cerveza es una de las bebidas alcohólicas más consumidas en todo el mundo, con una producción mundial anual estimada de 39 millones de toneladas (Lynch, et al., 2016). Esta industria utiliza como principal materia prima en su proceso cebada malteada, la cual es sometida a un proceso de cocción y maceración del que resulta el mosto cervecero, licor que luego continúa hacia la etapa de fermentación para la elaboración de la cerveza. En este proceso se producen cantidades 
importantes de residuo insoluble, conocido localmente como bagazo cervecero e internacionalmente como "Brewer's spent grain” (BSG) (Lynch, et al., 2016). Este subproducto representa el $85 \%$ de los residuos y es en promedio el $31 \%$ del peso original de malta utilizado durante el proceso (Nigam, 2017).

Actualmente, el destino más común dado por la industria para este subproducto es su disposición para alimentación animal, a pesar de ser una buena fuente de fibra, particularmente la insoluble (Buffington, 2014). El bagazo de cervecería es un material de alto valor, que contiene hemicelulosa, lignina y alto contenido de proteína (Fillaudeau, et al., 2006), monosacáridos de xilosa, glucosa y arabinosa, minerales y aminoácidos (Mussatto, 2009).

La gestión ambiental de los residuos tiene costo para todas las empresas. Además, en muchos casos se está perdiendo el costo oportunidad de su transformación en un ingrediente funcional con valor económico y nutricional para la industria alimentaria. La valorización de estos residuos representa una estrategia competitiva que abre la puerta a nuevas posibilidades de negocios de cara a la creciente demanda de alimentos funcionales.

Esta estrategia se encuentra alineada con los nuevos conceptos que han surgido en los últimos años de economía circular, que tiene como una de sus premisas la recuperación de materiales para que puedan ingresar en nuevos procesos de producción y llegar nuevamente a consumo (World Economic Forum, 2017). A su vez, encuentra otro punto de contacto con las políticas manifestadas por la Organización de las $\mathrm{Na}$ ciones Unidas para la Agricultura y la Alimentación de llegar a "pérdidas y desperdicios cero, hambre cero" (FAO, 2015).

Todo esto en su conjunto permite considerar que los residuos generados en el proceso de maceración de la malta son un subproducto con potencial en su valor nutricional y tecnológico. El bagazo de cervecería, o BSG, ha sido utilizado en la elaboración de productos como panes, galletas, muffins, tortas, snacks, entre otros (Lynch, et al., 2016; Mussatto, 2014), como ingrediente de productos funcionales (Kanauchi, et al., 2001; Ainsworth, et al., 2007; Ktenioudaki, et al., 2012; Fărcaş, et al., 2014). El pan presenta especial interés, ya que es un alimento muy consumido en todo el mundo y tiene el potencial para convertirse en un producto alimenticio funcional por su importante rol en la dieta humana diaria (Flander, et al., 2007). Los beneficios potenciales en la salud que la fibra dietaria genera han sido ampliamente estudiados y documentados, y se vinculan a la prevención del estreñimiento, la reducción en el riesgo de cáncer colorrectal y de enfermedades cardiovasculares, y la producción de ácidos grasos de cadena corta (Lattimer, et al., 2010; Zhu, et al., 2015).

Un aspecto que se debe contemplar si se espera que un producto llegue al mercado es el rotulado nutricional que le correspondería según la reglamentación vigente que aplique. En este aspecto, en el año 2018 se aprobó en Uruguay un Decreto sobre el etiquetado frontal (Uruguay, 2018), que prevé la inclusión de advertencias octogonales en el frente del empaque para el sodio, azúcares, grasas y grasas saturadas si los valores de estos ingredientes se encuentran por encima de los niveles establecidos en el decreto. Hasta el momento existe poca investigación sobre la percepción del etiquetado frontal y de otros tipos de etiquetado nutricional fuera de los Estados Unidos, Oceanía y Europa (Mandle, et al., 2015). Algunos estudios recientes sugieren que los consumidores tienden a elegir productos que no contienen advertencias en el etiquetado frontal frente a los que sí las incluyen (Arrúa, et al., 2017; Ares, et al., 2018a; Ares, et al., 2018b; Machín, et al., 2018). Como aún faltan evidencias de la influencia de este aspecto en las elecciones de los consumidores sobre productos reales, se entiende necesario tenerlo en cuenta para el desarrollo de nuevos productos y/o reformulación de existentes.

El objetivo de este trabajo fue desarrollar panes con incorporación de harina de bagazo de cervecería como nuevo ingrediente rico en fibra, evaluando su aceptabilidad por consumidores, y analizar el efecto de la información nutricional en el etiquetado frontal en la intención de compra y aceptabilidad de los productos desarrollados.

\section{Materiales y Métodos}

\section{Obtención de la harina de bagazo (BSG)}

El bagazo de cervecería fue provisto por la empresa FNC S.A. (Montevideo, Uruguay). Se secó a $50{ }^{\circ} \mathrm{C}$ en un horno de convección forzada hasta peso constante, se molió en un molino de laboratorio (Retsch ZM 200) y se tamizó con malla de $1 \mathrm{~mm}$, obteniéndose así la harina de bagazo de cervecería (BSG).

La BSG se utilizó como "aporte" de fibra en la formulación de panes enriquecidos según el procedimiento que se detalla a continuación.

\section{Elaboración de los panes}

Para la elaboración de los panes se utilizaron los siguientes ingredientes: harina de trigo, harina de bagazo de cervecería (BSG), leche en polvo descremada, azúcar, sal, levadura deshidratada, aceite y agua, según formulación establecida en Tabla 1 para el Ensayo 1 y Tabla 2 para el Ensayo 2.

Todos los ingredientes fueron dispuestos en el molde de pan de una panetera Punktal, siguiendo el programa de cocción para pan básico proporcionado por el equipo (temperatura de horneado $120^{\circ} \mathrm{C}$; tiempo de horneado $55 \mathrm{~min}$; tiempo total $3 \mathrm{hs}$ ). Los panes fueron desmoldados en caliente y se dejaron enfriar a temperatura ambiente. Se almacenaron en congelación $\left(-18^{\circ} \mathrm{C}\right)$, hasta 24 hs antes de su análisis, momento en el cual se dispusieron a temperatura ambiente.

\section{Ensayo 1}

Se consideró como variable el contenido de fibra a dos niveles, teniendo en cuenta el cumplimiento (Fórmula I) o no (Formula II) del claim "fuente de fibra". Se formuló además un pan de referencia, sin incorporación de fibra (Fórmula Referencia).

A la Fórmula I se le agregó harina de bagazo de cervecería (BSG) en la cantidad suficiente para que el pan resultante pudiera cumplir con el claim "fuente de fibra" en MERCOSUR (2012) (por lo menos 2,5 g de fibra dietaria por $50 \mathrm{~g}$ ). Este resultado se alcanzó sustituyendo un $15,6 \%$ de la harina de trigo por BSG, de acuerdo al contenido de fibra determinado en el BSG (44,6 $\mathrm{g}$ de fibra dietaria/100 $\mathrm{g}$ de harina de bagazo) (Tabla 3).

Para formular el pan II, enriquecido con BSG pero sin lo suficiente para cumplir el claim "fuente de fibra", se estableció sustituir un 7,8\% de harina de trigo por BSG (Tabla 1). En estudios preliminares se ensayaron diferentes incorporaciones 


\begin{tabular}{|l|c|c|c|}
\hline \multirow{2}{*}{ Ingredientes } & \multicolumn{2}{|c|}{ Cantidad (\%) } \\
\cline { 2 - 4 } & Pan Referencia & $\begin{array}{c}\text { Pan con BSG “fuente de fibra" } \\
\text { (Fórmula I) }\end{array}$ & Pan con BSG (Fórmula II) \\
\hline Agua & 33,37 & 33,37 & 33,37 \\
Harina de trigo & 53,30 & 45,00 & 49,15 \\
Harina de bagazo de cervecería (HBC) & - & 8,30 & 4,15 \\
Aceite de girasol & 4,86 & 4,86 & 4,86 \\
Azúcar & 3,60 & 3,60 & 3,60 \\
Leche en polvo & 3,20 & 3,20 & 3,20 \\
Sal & 1,20 & 1,20 & 1,20 \\
Levadura & 0,47 & 0,47 & 0,47 \\
\hline
\end{tabular}

Tabla 1. Formulación de los panes para el Ensayo 1.

\begin{tabular}{|l|c|c|c|c|}
\hline \multirow{2}{*}{ Ingredientes } & \multicolumn{4}{|c|}{ Cantidad (\%) } \\
\cline { 2 - 5 } & Formula I & Fórmula II & Fórmula III & Fórmula IV \\
\hline Agua & 33,37 & 33,37 & 33,77 & 33,77 \\
Harina de trigo & 45,00 & 49,15 & 45,55 & 49,75 \\
Harina de bagazo de cervecería (HBC) & 8,30 & 4,15 & 8,40 & 4,20 \\
Aceite de girasol & 4,86 & 4,86 & 4,92 & 4,92 \\
Azúcar & 3,60 & 3,60 & 3,64 & 3,64 \\
Leche en polvo & 3,20 & 3,20 & 3,24 & 3,24 \\
Sal & 1,20 & 1,20 & - & - \\
Levadura & 0,47 & 0,47 & 0,48 & 0,48 \\
Con claim “fuente de fibra" & SI & NO & SI & NO \\
Con advertencia "EXCESO DE SODIO" & SI & SI & NO & NO \\
\hline
\end{tabular}

Tabla 2. Formulación de los panes para el Ensayo 2.

de BSG a los panes (por debajo del mínimo para alcanzar el claim "fuente de fibra") y se halló que una sustitución del $7,8 \%$ de harina de trigo por BSG era adecuada para obtener un pan que fuera aceptado por los consumidores (datos no mostrados)

\section{Ensayo 2}

A las formulaciones establecidas en el Ensayo 1 se les incorpora como variable el contenido de Sodio, para contemplar la advertencia respecto al ingrediente ("EXCESO DE SODIO" o sin advertencia), según el Decreto sobre etiquetado frontal 272/018 (Uruguay, 2018) aprobado en agosto del 2018 en Uruguay. En este caso, resultaron cuatro fórmulas de pan (I, II, III y IV). Por un lado, las fórmulas I y II desarrolladas en el Ensayo 1, con un contenido de sal usualmente encontrado en panes del mercado uruguayo, que resultan en niveles de Sodio por encima de lo establecido en el Decreto de Etiquetado
Frontal, y por tanto deberían incluir en el rotulado la advertencia “EXCESO DE SODIO". Por otro lado, las fórmulas III y IV, que representan las formulaciones I y II pero sin agregado de sal, con niveles de Sodio por debajo de lo establecido en el Decreto, y que, por tanto, no deben incluir la advertencia en cuestión (Tabla 2).

\section{Composición nutricional}

Se determinó la composición nutricional de la harina de bagazo de cervecería. El contenido de proteína y de fibra dietaria total (FDT) se determinaron de acuerdo a los métodos de la AOAC 984.13 (AOAC International, 2012a) y 985.29 (AOAC International, 2012b), respectivamente. El contenido graso se estimó de acuerdo a la ISO 6492 (International Organization for Standarization, 1999). El contenido de humedad se determinó por análisis gravimétrico en estufa a $105^{\circ} \mathrm{C}$ hasta peso constante. Las cenizas se 
determinaron por incineración en mufla, de acuerdo a la ISO 5984 (International Organization for Standarization, 2002, cor.1 2005). El contenido de carbohidratos totales se determinó por diferencia entre el peso total y la suma de los gramos de proteínas, lípidos, fibra dietaria, humedad y cenizas contenidas en 100 gramos de muestra. Se determinó además el contenido de sodio, de acuerdo al método de la AOAC 2011.14 (AOAC International, 2016).

\section{Evaluación sensorial por consumidores}

\section{Ensayo 1}

Los panes elaborados en el Ensayo 1 (Tabla 1) fueron evaluados por 77 consumidores, estudiantes y funcionarios de la Universidad Católica del Uruguay y del Parque Tecnológico del LATU, consumidores habituales de pan, $48 \%$ hombres y $52 \%$ mujeres, con edades entre los 18 y 69 años. Una rodaja de cada pan fue servida en platos codificados con números aleatorios de tres dígitos y entregados a cada participante para su evaluación. Los participantes fueron invitados a probar cada muestra de pan e indicar su agrado general utilizando una escala hedónica de 9 puntos (1: "No me gusta en absoluto" a 9: "Me gusta muchísimo).

\section{Ensayo 2}

Para evaluar el efecto de la información nutricional presentada en el etiquetado frontal, se realizó una segunda evaluación con consumidores, quienes evaluaron los panes elaborados en el Ensayo 2 (Tabla 2). En esta oportunidad se presentó a los consumidores una rodaja de cada pan formulado junto con su posible etiqueta. Participaron en esta etapa 81 consumidores, estudiantes y funcionarios de la Universidad Católica del Uruguay y del Parque Tecnológico del LATU, consumidores habituales de pan, $48 \%$ hombres y $52 \%$ mujeres, con edades entre los 18 y 65 años. Una rodaja de cada pan fue servida en platos codificados con números aleatorios de tres dígitos y entregados a cada participante para su evaluación junto con su etiqueta correspondiente. Los participantes fueron invitados a evaluar cada etiqueta indicando su intención de compra (1:"Definitivamente no lo compraría" a 7:"Definitivamente lo compraría") y probar cada muestra de pan indicando su agrado general utilizando una escala hedónica de 9 puntos (1: "No me gusta en absoluto" a 9: "Me gusta muchísimo).

\section{Diseño de etiquetas}

Se diseñaron etiquetas de los panes formulados con la información nutricional que le corresponde a cada formulación en cuestión, considerando como variables la inclusión o no del claim "fuente de fibra" y la advertencia o no de "EXCESO DE SODIO”. Para esta última se tomó el diseño octogonal de fondo negro y borde blanco, con la expresión en su interior "EXCESO DE SODIO", como establece el Decreto sobre etiquetado frontal 272/018 (Uruguay, 2018), y su tamaño se estableció de acuerdo a las especificaciones establecidas.

Las etiquetas no corresponden a ningún producto en particular disponible en el mercado uruguayo de modo de evitar la influencia en la respuesta de los consumidores. Las etiquetas fueron diseñadas por una empresa de diseño gráfico con experiencia en el diseño de envases de alimentos. En la Figura 1 se presenta una imagen de las etiquetas diseñadas.
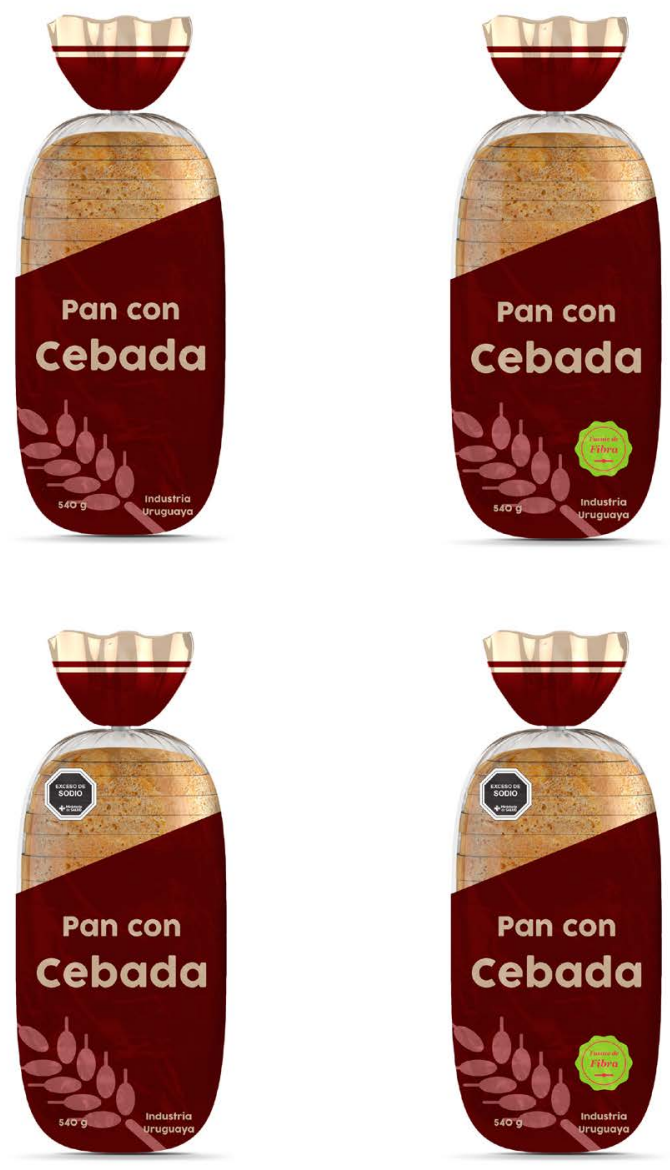

Figura 1. Imagen de las etiquetas diseñadas presentadas en la evaluación sensorial realizada para el Ensayo 2.

\section{Análisis estadístico}

El Análisis de Varianza (ANOVA) de un factor fue utilizado para analizar los datos de la aceptabilidad de las muestras elaboradas para el Ensayo 1 (productos sin información) sobre los datos de aceptabilidad e intención de compra para el Ensayo 2 (productos con etiqueta). El ANOVA de dos factores fue utilizado para evaluar el efecto de la fibra (enriquecido; fuente de fibra) y del contenido de sal (con sal; sin sal) sobre la aceptabilidad e intención de compra para las muestras del Ensayo 2. Las diferencias significativas entre muestras fueron determinadas con el test de Tukey $(\mathrm{p} \leq 0,05)$.

Los análisis fueron ejecutados usando XLSTAT Versión 2011 (Addinsoft 1995-2010, France).

\section{Resultados y Discusión}

\section{Composición proximal de la harina de bagazo de cervecería}

Se determinó la composición proximal de la harina de bagazo de cervecería (BSG), incluyendo la estimación de proteínas, materia grasa, cenizas, fibra dietaria y carbohidratos y sodio, parámetros requeridos para el rotulado nutricional de los productos envasados (Tabla 3 ). 


\begin{tabular}{|l|c|}
\hline & Harina de bagazo de cervecería \\
\hline Proteínas & $23,81 \pm 0,31$ \\
Lípidos & $6,24 \pm 0,01$ \\
Cenizas & $3,76 \pm 0,06$ \\
Fibra dietaria & $44,61 \pm 0,29$ \\
Carbohidratos & $14,89^{1}$ \\
Sodio $^{2}$ & 240 \\
\hline
\end{tabular}

1. Contenido de carbohidratos determinado por diferencia.

2- Contenido de sodio expresado en $\mathrm{mg} / \mathrm{kg}$

Tabla 3. Composición proximal de la harina de bagazo de cervecería en $\mathrm{g} / 100 \mathrm{~g}$.

Los valores obtenidos en este trabajo concuerdan con los informados por Aliyu y Bala (2010), quienes reportan para el BSG valores de proteínas entre un $15-26 \%$, materia grasa $3,9-10 \%$ y fibra dietaria entre $50-70 \%$.

Ktenioudaki et al. (2012) evaluaron el BSG por su potencial como ingrediente funcional en productos horneados y mostraron que la adición de un 25-35\% aumentaba significativamente el contenido de proteínas de snacks y que la adición del 15\% duplica el contenido de fibra. Este resultado reafirma el potencial que presenta la harina de bagazo como ingrediente funcional, fuente de fibras y proteínas, y su posible incorporación a alimentos para consumo humano, dándole un mayor valor agregado que el derivado de su uso más frecuente en alimentación animal.

\section{Desarrollo de panes enriquecidos con harina de bagazo de cervecería}

Se realizó una evaluación sensorial con consumidores, quienes evaluaron la aceptabilidad de panes con incorporación de BSG (fórmulas I y II) y de un pan referencia sin incorporación de BSG. Los valores medios de aceptabilidad obtenidos para cada pan se presentan en la Tabla 4.

De acuerdo al ANOVA, la aceptabilidad del pan enriquecido con BSG (sustitución del 4,15\% de BSG en la formulación total) no presenta diferencia significativa con el pan sin BSG (pan referencia), y ambos son significativamente más aceptables que el pan "fuente de fibra" (sustitución del 8,30\% de BSG en la formulación total) ( $p$ valor $<0,0001$ ).

\begin{tabular}{|l|c|}
\hline & Aceptabilidad \\
\hline Pan con BSG “fuente de fibra" (fórmula 1) & $5,5^{\mathrm{a}}$ \\
\hline Pan con BSG (fórmula II) & $6,5^{\mathrm{b}}$ \\
Pan Referencia & $6,8^{\mathrm{b}}$ \\
\hline
\end{tabular}

Letras diferentes implican diferencias significativas entre muestras $(\mathrm{p}<0.05)$.

Tabla 4. Valor medio de aceptabilidad para los panes del Ensayo 1.

Ktenioudaki et al. (2013) obtuvieron resultados similares al estudiar la incorporación de BSG en panes, y lograron productos aceptables con una incorporación de un 10\% de BSG en la fórmula total. Por encima de este porcentaje, la aceptabilidad disminuyó significativamente, generando rechazo en el consumidor.

El BSG demuestra ser una buena alternativa como ingrediente funcional, debido a que se pueden formular panes con $1,25 \%$ de fibra dietaria (Fórmula II) sin afectar significativamente la aceptabilidad. Para formular panes con 2,5\% de fibra dietaria, y así denominarlos como "fuente de fibra" (Fórmula 1), se deberían realizar cambios más significativos en la formulación.

\section{Efecto de la información nutricional en la aceptabilidad e intención de compra}

Para evaluar el impacto de la información nutricional en la intención de compra y la aceptabilidad de los panes formulados, se consideró evaluar como variable el contenido de sodio, además del contenido de fibra. La variable sodio se plantea porque, en general, la mayoría de los panes envasados disponibles en el mercado presentan un contenido de sodio por encima del límite que establece el Decreto de Rotulado Frontal, y por tanto deberían presentar una advertencia por "EXCESO DE SODIO". Se evaluaron los panes con incorporación de BSG y con el contenido de sal usualmente encontrado en los panes del mercado uruguayo, y las mismas formulaciones pero sin incorporación de sal.

Como muestra la Tabla 5, la intención de compra y la aceptabilidad variaron significativamente entre las cuatro formulaciones estudiadas para el Ensayo 2.

\begin{tabular}{|l|c|c|}
\hline & $\begin{array}{c}\text { Intención } \\
\text { de compra }\end{array}$ & Aceptabilidad \\
\hline $\begin{array}{l}\text { Pan con BSG "fuente de } \\
\text { fibra" con sal (Fórmula I) }\end{array}$ & $4,4^{\mathrm{b}, \mathrm{c}}$ & $5,9^{\mathrm{a}, \mathrm{b}}$ \\
$\begin{array}{l}\text { Pan con BSG con sal } \\
\text { (Fórmula II) }\end{array}$ & $3,8^{\mathrm{c}}$ & $6,6^{\mathrm{a}}$ \\
$\begin{array}{l}\text { Pan con BSG "fuente de } \\
\text { fibra" sin sal (Fórmula III) }\end{array}$ & $5,4^{\mathrm{a}}$ & $4,5^{\mathrm{c}}$ \\
$\begin{array}{l}\text { Pan con BSG sin sal } \\
\text { (Fórmula IV ) }\end{array}$ & $4,7^{\mathrm{b}}$ & $5,1^{\mathrm{b}, \mathrm{c}}$ \\
\hline
\end{tabular}

Letras diferentes en una misma columna implican diferencias significativas entre muestras $(\mathrm{p}<0,05)$.

Tabla 5. Valor medio de intención de compra y aceptabilidad para los panes del Ensayo 2.

En lo que a la información respecta, los consumidores prefirieron el pan "fuente de fibra" y sin sal frente al resto de las formulaciones y rechazaron el pan enriquecido con fibra con sal. Los panes "fuente de fibra" con sal y el pan enriquecido con fibra sin sal tuvieron intención de compra intermedia (escala utilizada para intención de compra 1-7).

Esto lleva a pensar que la información respecto al contenido de sal y fibra tuvo un impacto real en la elección de los consumidores. Para evaluarlo, se estudió el impacto del efecto fibra y el efecto sal independientemente mediante una ANOVA de dos factores. Como se observa en la Tabla 6, ambos efectos, sal y fibra, fueron significativos tanto para la 
intención de compra como para la aceptabilidad. Sin embargo, la interacción no lo fue.

\begin{tabular}{|l|c|c|}
\hline & Intención de compra & Aceptabilidad \\
\hline Sal & $<0,0001$ & $<0,0001$ \\
Fibra & 0,0005 & 0,0020 \\
SalFibra & 0,6422 & 0,8411 \\
\hline
\end{tabular}

Tabla 6. p-valor de los efectos fijos utilizados para el ANOVA de dos factores (sal y fibra) sobre la intención de compra y la aceptabilidad para los panes del Ensayo 2.

Por lo tanto, ambos efectos son percibidos de forma independiente por el consumidor, la advertencia "EXCESO DE SODIO" tiene un impacto negativo y la presencia del claim "fuente de fibra" tiene un impacto positivo (Tabla 5).

De acuerdo a los resultados obtenidos en este trabajo, la presencia de la frase "EXCESO DE SODIO" presentada en el envase provocaría una disminución en la intención de compra de los consumidores. Por lo menos, para este componente y en este tipo de alimento, se estaría cumpliendo con el propósito que tiene la implementación de este sistema de advertencias, que es desalentar el consumo de productos considerados como no saludables (Corvalán, et. al., 2013; Chile, 2015). Este sistema de octógonos basado en advertencias sobre cierto tipo de nutrientes o componentes, se implementa en Chile desde el año 2015.

Machín et al. (2018) obtuvieron resultados similares al evaluar el impacto de la presencia de diferentes sistemas de advertencia sobre la intención de compra de los consumidores. En su estudio, los consumidores fueron invitados a imaginar que debían comprar productos para preparar un almuerzo saludable. En este trabajo, hipotéticamente los uruguayos consumirían un 19\% menos de sodio en su dieta si los alimentos estuvieran rotulados con los octógonos. De la misma forma, Ares et al. (2018b), focalizando el estudio en tres categorías de productos y también de modo teórico, encontraron que para panes, yogures y queso crema los uruguayos prefirieron los productos sin advertencias frente a los productos con advertencias.

Se debería ampliar la investigación a otras categorías de productos con evaluaciones prácticas y en distintos segmentos de la población, para evaluar el efecto de este sistema de rotulado en la aceptación de los consumidores uruguayos por los alimentos que componen su dieta.

$\mathrm{Al}$ evaluar los panes sensorialmente y valorar la aceptabilidad, el pan enriquecido y con sal fue el preferido y el pan "fuente de fibra" sin sal fue el menos aceptado por los consumidores (Tabla 5). Al evaluar los efectos por separado en ANOVA de dos factores (Tabla 6), tanto el efecto fibra como el efecto contenido de sal fueron significativos.

El pan con mayor intención de compra inicial (fórmula III) fue el que obtuvo menor aceptabilidad sensorial, mientras que el pan con menor intención de compra inicial fue el que obtuvo mayor aceptabilidad sensorial.

Si bien el consumidor compraría el pan "fuente de fibra" y sin advertencia de sal, luego al probarlo lo rechazaría (valor de aceptabilidad menor a 5), lo cual indica que sería oportuno realizar esfuerzos y trabajar en la reformulación de los productos para alcanzar productos aceptables y cumplir con el propósito del rotulado con octógonos.

\section{Conclusiones}

El subproducto generado por la industria cervecera, BSG, es factible de ser utilizado como ingrediente funcional. Se destacan como componentes más relevantes un contenido de fibra dietaria de $44,6 \%$ y proteínas del $23,8 \%$.

La incorporación de fibra en la formulación de panes podría ser una buena alternativa para mejorar la aceptación de fórmulas reducidas en sal.

La presencia de la advertencia "EXCESO DE SODIO" representada en el envase disminuye la intención de compra de los consumidores por panes con cebada con dicho mensaje, mientras que la presencia del claim "fuente de fibra" aumenta la intención de compra de los panes que la contengan.

Si bien la información determina la elección inicial de los consumidores por los panes, el producto será rechazado si no alcanza valores de aceptabilidad sensorial favorables. Es una oportunidad para la industria, que debería focalizar sus esfuerzos hacia la reformulación y/o desarrollo de productos aceptables y sin mensajes percibidos como negativos por los consumidores.

Este trabajo demuestra que, a través de la reformulación de alimentos con ingredientes beneficiosos para la salud de los consumidores como el BSG, se puede dar solución a la industria para alcanzar productos aceptables manteniendo una adecuada intención de compra.

\section{Reconocimientos}

A FNC S.A. por proveernos del bagazo para todos los ensayos. A Syrah Comunicación Visual por el diseño de las etiquetas.

\section{Referencias}

Ainsworth, Ibanoglu, S., Plunkett, A., Ibanoglu, E. y Stojceska, V., 2007. Effect of brewers spent grain addition and screw speed on the selected physical and nutritional properties of an extruded snack. En: J. Food Eng., 81, pp.702-709.

Aliyu, S. y Bala, M., 2010. Brewer's spent grain: a review of its potentials and applications. En: Afr. J. Biotechnol., 10, pp.324-331.

AOAC International, 2012a. Official methods of analysis of AOAC international. 19a ed. Gaithersburg: AOAC. Official Method 984.13, first action 1984-final action 1994.

AOAC International, 2012b. Official methods of analysis of AOAC international. 19a ed. Gaithersburg: AOAC. Official Method 985.29, first Action 1985-final action 1986.

AOAC International, 2016. Official methods of analysis of AOAC international. 20a ed. Gaithersburg: AOAC. Official Method 2011.14, first action 2011-final action 2013.

Ares, G., Aschemann-Witzel, J., Curutchet, M.R., Antúnez, L., Machín, L. y Vidal, L., 2018a. Nutritional warnings and product substitution or abandonment: policy implications derived from a repeated purchase simulation. En: Food Quality and Preference, 65, pp.40-48. https://doi. org/10.1016/j.foodqual.2017.12.001

Ares, G., Aschemann-Witzel, J., Curutchet, M.R., Antúnez, L., Machín, L., Vidal, L. y Giménez, A., 2018b. Product reformulation in the context of nutritional warning labels: exploration of consumer preferences towards food concepts in three food categories. En: Food Research 
International, 107, pp.669-774. https://doi:10.1016/j. foodres.2018.03.021

Arrúa, A., Curutchet, M.R., Rey, N., Barreto, P., Golovchenko, N., Sellanes, A., Velazco, G., Winokur, M., Giménez, A. y Ares, G., 2017. Impact of front-of-pack nutrition information and label design on children's choice of two snack foods: Comparison of warnings and the traffic-light system. En: Appetite, 116, pp.139-146.

Buffington, J., 2014. The economic potential of brewer's spent grain (BSG) as a biomass feedstock. En: Adv. Chem. Eng. Sci., 4, pp.308-318.

Chile. Decreto 13/15, de 16 de abril de 2015. Diario Oficial, 26 de junio de 2015. [En línea]. [Consulta: setiembre 2017] Disponible en: http://www.wipo.int/edocs/lexdocs/laws/ es/cl/cl074es.pdf

Corvalán, C., Reyes, M., Garmendia, M.L. y Uauy, R. 2013. Structural responses to the obesity and non-communicable disease epidemic: The Chilean law of food labelling and advertising. En: Obesity Reviews, 14, pp.79-87.

FAO. Organización para las Naciones Unidas para la alimentación y la Agricultura, 2015. Pérdidas y desperdicios de alimentos en América Latina y el Caribe. Roma: FAO. (Boletín; 2).

Fărcaş, A., Tofană, M., Socaci, S., Mudura, E., Scrob, S., Salanță, L. y Mureşan, V., 2014. Brewers' spent grain - A new potential ingredient for functional foods. En: Journal of Agroalimentary Processes and Technologies, 20(2), pp.137-141.

Fillaudeau, L., Blanpain-Avet, P. y Daufin, G., 2006. Water, wastewater and waste management in brewing industries. En: Journal of Cleaner Production, 14, pp.463-471.

Flander, L., Salmenkallio-Marttila, M., Suortti, T. y Autio, K., 2007. Optimization of ingredients and baking process for improved wholemeal oat bread quality. En: $L W T-$ Lebensmittel-wissenschaft \& Technologie, 40, pp.860-870.

Kanauchi, O., Mitsuyama, K. y Araki, Y., 2001. Development of a functional germinated barley foodstuff from brewers' spent grain for the treatment of ulcerative colitis. En: J. Am. Soc. Brewing Chemists, 59, pp.59-62.

International Organization for Standarization, 1999. ISO 6492: Animal feeding stuffs -- determination of fat content. Ginebra: ISO.

International Organization for Standarization, 2002 cor.1 2005. ISO 5984: Animal feeding stuffs -- Determination of crude ash. Ginebra: ISO.

Ktenioudaki, A., Chaurin, V., Reis, S.F. y Gallagher, E., 2012. Brewer's spent grain as a functional ingredient for breadsticks. En: Int. J. Food Sci. Tech., 47, pp.1765-1771.
Ktenioudaki, A., Crofton, E., Scannell, A.G.M., Hannon, J.A., Kilcawley, K.N. y Gallaghera, E., 2013. Sensory properties and aromatic composition of baked snacks containing brewer's spent grain. En: J. Cereal Sci., 57, pp.384-390.

Lattimer, J.M. y Haub, M.D., 2010. Effects of dietary fiber and its components on metabolic health. En: Nutrients, 2, pp.1266-89.

Lynch, K.M., Steffen, E.J. y Arendt, E.K., 2016. Brewers' spent grain: a review with an emphasis on food and health. En: $J$. Inst. Brew., 122, pp.553-568.

Machín, L., Aschemann-Witzel, J., Curutchet, M.R., Giménez, A. y Ares G., 2018. Does front-of-pack nutrition information improve consumer ability to make healthful choices? Performance of warnings and the traffic light system in a simulated shopping experiment. En: Appetite, 121, pp.55-62.

Mandle, J., Tugendhaft, A., Michalow, J. y Hofman, K., 2015. Nutrition labelling: a review of research on consumer and industry response in the global south. En: Global Health Action, 8(1), pp.25912-10.

MERCOSUR, 2012. MERCOSUR/GMC/RES.

$N^{\circ} 01 / 12$. Reglamento técnico Mercosur sobre información nutricional complementaria (declaraciones de propiedades nutricionales) [En línea]. Buenos Aires: GMC.

[Consulta: agosto de 2017]. Disponible en: http://www.montevideo.gub.uy/sites/default/files/ resolucion mercosur 1 2012.pdf

Mussatto, S. I., 2009. Biotechnological potential of brewing industry by-Products. En: Singh-Nee Nigam, Poonam, Pandey, Ashok, (eds.). Biotechnology for agroindustrial residues utilization. Berlín: Springer, pp.313-326.

Mussatto, S.I., 2014. Brewer's spent grain: a valuable feedstock for industrial applications. En: J. Sci. Food Agric., 94, pp.1264-127.

Nigam, P.S., 2017. An overview: Recycling of solid barley waste generated as a by-product in distillery and brewery. En: Waste Management, 62, pp.255-261.

Uruguay. Decreto 272/018, de 29 de agosto de 2018. Diario Oficial, 31 de agosto de 2018, No. 30.018, p.10.

World Economic Forum, 2017. From linear to circularaccelerating a proven concept [En línea]. Ginebra: World Economic Forum. [Consulta: 3 de febrero de 2018]. Disponible en: http://reports.weforum.org/toward-thecircular-economy-accelerating-the-scale-up-across-globalsupply-chains/from-linear-to-circular-accelerating-aproven-concept/

Zhu, F., Du, B., Zheng, L. y Li, J., 2015. Advance on the bioactivity and potential applications of dietary fibre from grape pomace. En: Food Chemistry, 12, pp.186-207. 